\title{
Review Article \\ Identifying and Overcoming Perceived Barriers of Providers towards HPV Vaccination: A Literature Review
}

\author{
Jessica Palmer, Catherine Carrico, and Cindy Costanzo \\ Creighton University, 2500 California Plaza, Omaha, NE 68178, USA \\ Correspondence should be addressed to Catherine Carrico; cathycarrico@creighton.edu
}

Received 31 August 2015; Revised 12 November 2015; Accepted 19 November 2015

Academic Editor: Itabajara da Silva Vaz Jr

Copyright (C) 2015 Jessica Palmer et al. This is an open access article distributed under the Creative Commons Attribution License, which permits unrestricted use, distribution, and reproduction in any medium, provided the original work is properly cited.

Human papillomavirus (HPV) is a common sexually transmitted infection in the United States associated with nearly 26,000 cases of cancer annually. With the recent addition of Gardasil 9, three vaccines are now licensed by the Food and Drug Administration (FDA) and recommended by the Advisory Committee on Immunization Practices (ACIP) for HPV prevention. While providers are the preferred source in educating individuals on HPV and HPV vaccination, low uptake percentages indicate that providers are missing valuable opportunities to educate and strongly recommend HPV vaccination. It is critical to examine perceived barriers and attitudes among providers related to HPV and HPV vaccination to identify factors that influence vaccination coverage. This paper aims to expand provider knowledge and awareness of factors that may facilitate an increase in HPV vaccination coverage and subsequent cancer prevention.

\section{Introduction}

Human papillomavirus (HPV) currently infects 79 million Americans with 14 million newly infected each year and is the most common sexually transmitted infection in the United States (US) [1]. While most adults who are sexually active will become infected at some point in their lives, fifty percent of new infections occur among 15-24-year-olds [1].

Each year over 600,000 cases of cancer worldwide and 26,900 new cases of anal, cervical, oropharyngeal, penile, vaginal, and vulvar cancer in the US are attributed to HPV with cervical and oropharyngeal cancers being the most common causes of HPV-associated deaths [2,3]. The prevalence rate of $\mathrm{HPV}$-associated oropharyngeal cancers has increased from $16.3 \%$ in the 1980 s to $72.7 \%$ in the 2000 s [4]. In addition, HPV causes more than 300,000 cases of genital warts and 820 cases of recurrent respiratory papillomatosis (RRP) annually [2]. The economic burden of HPV-associated disease exceeds that of all other sexually transmitted infections except HIV with the annual direct medical costs for prevention and treatment tallying $\$ 8$ billion $[5,6]$.

Currently three HPV vaccinations are licensed by the Food and Drug Administration (FDA) and recommended by the Advisory Committee on Immunization Practices (ACIP) for protection against specific types of HPV for persons aged 11-26 years [7]. Unfortunately, national uptake percentages among the targeted population of vaccine-eligible individuals show that only $37.6 \%$ of females and $13.9 \%$ of males aged 13-17 years have received the 3-dose HPV vaccination series suggesting that providers are missing valuable opportunities to strongly recommend HPV vaccination (Figure 1) [8].

Up to $87 \%$ of girls and boys aged 11-17 years have had a missed opportunity for HPV vaccination within the preceding 12 months $[9,10]$. The greatest percentage of missed opportunities, $94 \%$, is associated with nonpreventive visits [11].

The need to encourage stronger provider recommendations for vaccination with the HPV series and utilize effective communication strategies for consumers in order to reduce the number of missed clinical opportunities for HPV vaccination was identified in The President's Cancer Panel Annual Report, 2012-2013 [3]. The American Academy of Pediatrics (AAP) [12], American Academy of Family Physicians (AAFP), American College of Physicians (ACP), and the American College of Obstetricians and Gynecologists (ACOG) in conjunction with the Immunization Action Coalition and the Centers for Disease Control and Prevention (CDC) also responded by establishing guidelines for education and HPV vaccination. Health People 2020 has set 


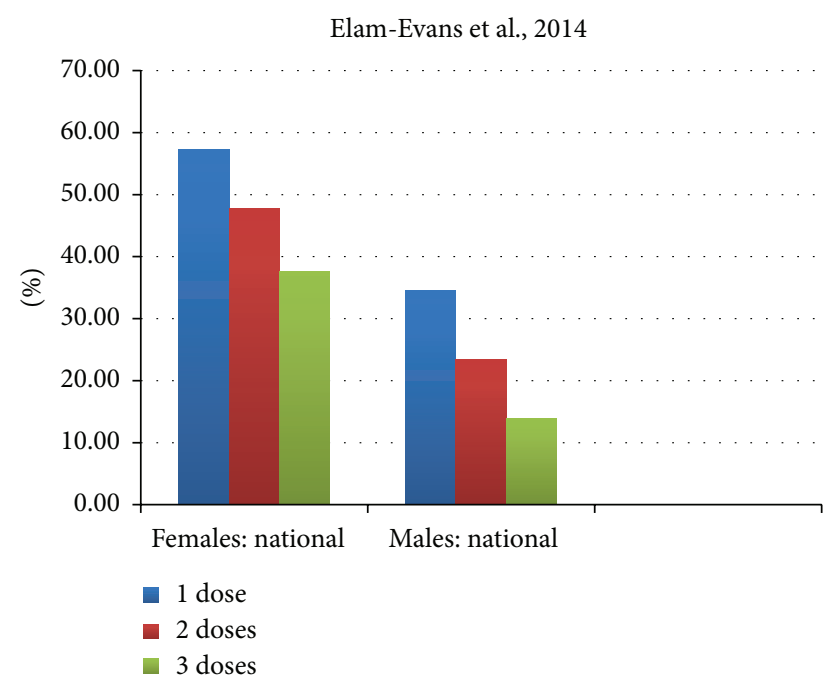

FIGURE 1: HPV vaccination coverage.

a goal of vaccine series completion for both females and males aged $13-15$ years to $80 \%$ by the year 2020 [13]. This literature review examines the evidence on the perceived barriers and attitudes of providers towards HPV vaccination, despite the recommendations of many organizations (Figure 2).

The review will then provide a comprehensive review of HPV and HPV vaccination and explore strategies that may facilitate an increase in HPV vaccination coverage and subsequent cancer prevention for adolescents. These strategies include the need for providers to utilize every patient encounter to address immunization status, coadministration of HPV vaccination with other routine adolescent vaccinations, the provision of vaccine information that promotes consumer awareness, discussing the need to return for the remaining doses in the HPV series, emphasis on vaccine benefit and cancer prevention, and provider knowledge.

\section{Perceived Barriers to Vaccination among Providers}

Healthcare providers have been identified as the most preferred, trusted source for medical information with their recommendation being one of the most important determinants of vaccination. Perceived barriers to vaccination can contribute to a lack of provider recommendation, missed opportunities for vaccination, and low rates of vaccination coverage among adolescents [14-16]. The barriers are categorized and discussed as follows: knowledge, vaccine safety, cultural, policy, cost, and structural barriers to vaccine [17, $18]$.

2.1. Knowledge. Knowledge barriers are significant, as a limited knowledge of HPV, including its prevalence, health implications, and vaccine efficacy, was found among providers and can affect the provider's ability to confidently deliver patient education [18-20]. A 2010 study identified only $58 \%$ of family physicians, and $43 \%$ of pediatricians knew that a difference existed among the HPV types that cause genital warts and those that cause cervical cancer [18].

Providers have also noted ineffective interpersonal and communication skills as well as knowledge of strategies for education of hesitant families and vaccine series completion $[18,21]$. For example, discussing sexuality prior to recommendation of vaccine was felt necessary in $42 \%$ of pediatricians, and $54 \%$ of family physicians [18], whereas others are more comfortable with discussion of sexuality in the older adolescent $[18,19]$. With providers serving as influential sources of medical information for patients, the identification of these barriers stresses the need for providers to be knowledgeable of HPV disease and current HPV vaccines and policy recommendations due to the expectation of accurately communicating such information to patients.

2.2. Safety. Providers have reported the need for more safety and efficacy vaccine information in order to recommend the vaccine, although they themselves were not overly concerned with regard to safety. A large number of providers report perceptions of parent concerns for safety as a barrier [18, $19,22]$. Parental fear of side effects and the vaccine being dangerous to their child have been cited in the literature, with higher concern if the child is 9-12 (46.3\%) versus 13-18 (41.4) years of age $[19,23]$.

2.3. Culture. Cultural barriers for providers and parents include myths contributing to negative perceptions towards HPV vaccination and moral or religious beliefs [19]. A key existing myth is the concern of HPV vaccination encouraging sexual promiscuity $[19,24]$. Likewise, the perceived young age of the patient in regard to receiving a vaccine for an infection that may be sexually transmitted was also a providerperceived barrier $[19,24]$. Studies have indicated that a range of $50 \%-89 \%$ of providers strongly recommended and administer vaccination for 11-12-year-olds, with most waiting until 13 years or older [18, 19]. Such factors as considering it necessary to discuss sexuality at an early age and parental refusals to vaccinate younger patients were associated with not strongly recommending HPV vaccination to 11-12-yearolds [18]. A provider's own personal estimation of a patient's sexual debut or activity despite their admittedly limited ability to accurately do so often leads to a deprioritization and delay of vaccination or nonvaccination [15]. Other moral or religious beliefs of the parent can delay HPV vaccination [19]. Gender issues also exist, with vaccination more routinely recommended for girls (76\%) as compared to boys (46\%) regardless of age [18].

2.4. Cost and Policy. Policy and costs barriers are closely related. Costs associated barriers are frequently reported as a barrier for providers, including concerns of lack of insurance coverage, lack of adequate reimbursement for HPV vaccination, and vaccine purchasing cost [17-19, 24, 25]. In addition, parents' out-of-pocket costs were a concern for providers [17].

National and international policy recommendations for $\mathrm{HPV}$ vaccine include the Centers for Disease Control and 


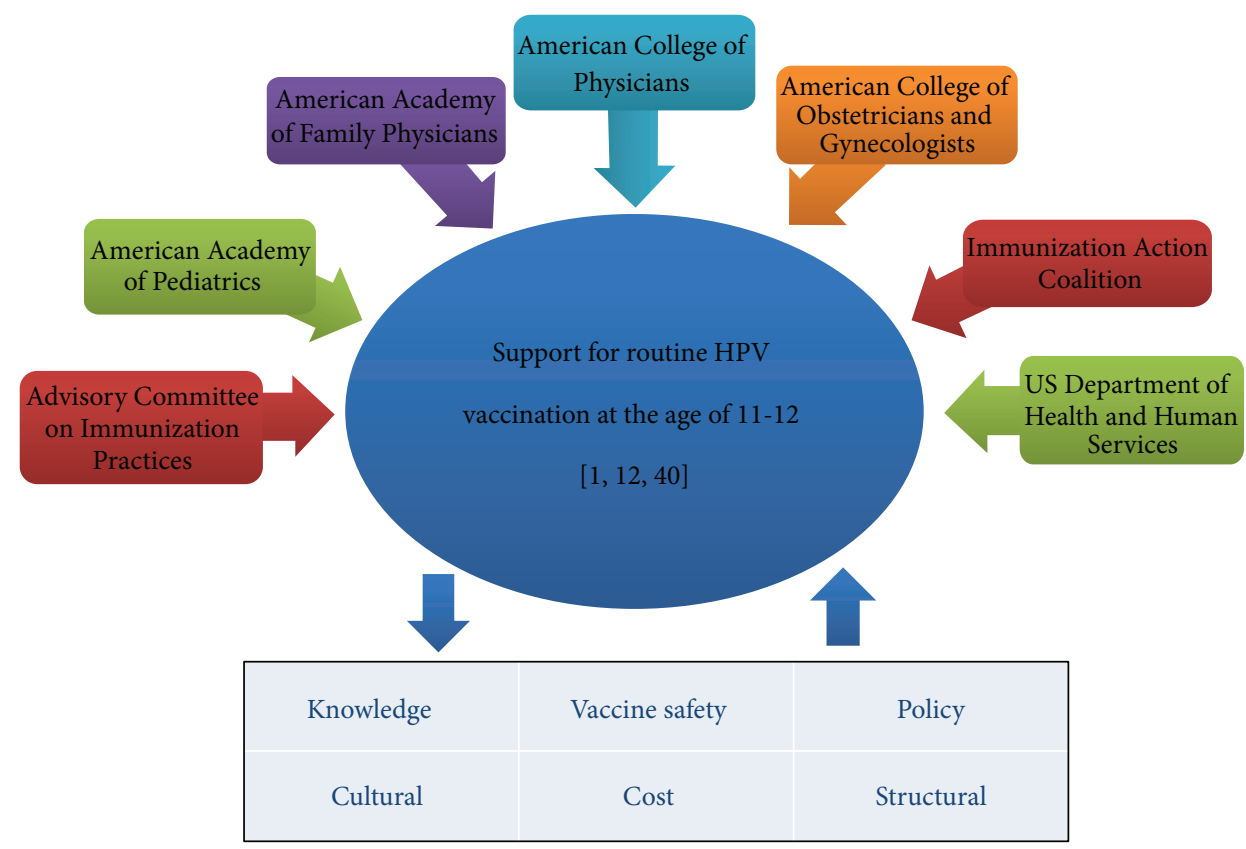

Figure 2

Prevention (CDC), ACIP, Healthy People 2020, and the World Health Organization (WHO). Each has stated goals and recommendations for vaccination and series completion. However, policy barriers for HPV vaccination can exist at the district, local, or state level. School mandates can be very effective for increasing vaccination coverage for required vaccines [26]; however a decline in routine preventative visits among adolescents past the 7 th grade is a continued problem. While concerns of inconsistent annual and well visits are higher for those working in public clinics, private practice providers cited more parental resistance and hesitancy to young vaccination age and coupling the vaccine with other recommended vaccinations than providers at public clinics [15]. Additional policy related barriers include need for written parental consent and tracking of immunization records, either via paper or through a vaccination registry [27].

2.5. Structural. Structural barriers include inadequate infrastructure within the primary care setting that can augment the delay in vaccination. Children in the targeted age group are often generally seen for urgent-care appointments and parents are hesitant to vaccinate while they are currently ill, despite no contraindication to vaccination $[7,17,22]$. Providers also report parental vaccine safety concerns and not having adequate time to comprehensively discuss these concerns during visits as a barrier $[19,22]$. The three-dose series requires additional return visits, and a lack of scheduled reminders and reminder strategies from the office can lead to parents forgetting to return with their adolescent for subsequent doses [19]. Although alternative sites for vaccine administration have been reported, they are not without their own barriers. One example is the use of school based health centers, which offers the convenience of a health provider while the child is in the school setting. However, there are only approximately 2000 such centers across the US [28]. Pharmacy barriers include a variance in the US state laws ruling the ability of the pharmacist to offer and administer the vaccine. In $80 \%$ of the states, pharmacists may provide HPV vaccine only to women 19 years and older, $61 \%$ to those of ages 12 years and older, most of which required prior physician approval or standing orders [29].

\section{Strategies for Breaking Down Barriers}

The identified barriers in the literature for providers can be barriers both independent of and dependent on one another. It is important to review the strategies to remove these barriers.

3.1. Knowledge of HPV. Studies have identified that providers, especially pediatricians and family practitioners who are most likely to administer the HPV vaccine to adolescents, would likely benefit from education clarifying which types of cancers the HPV vaccine prevents, the potential health implications associated with type-specific HPV infection, and the protection offered by the vaccine [2]. The following will address the HPV virus: the vaccine, the transmission, and the outcomes of these diseases.

While over $100 \mathrm{HPV}$ types have been identified, 40 types are divided into low-risk or nononcogenic serotypes and high-risk or oncogenic types [5]. Infection with the nononcogenic types, including types 6 and 11, is associated with low-grade cervical cell abnormalities and $90 \%$ of genital warts and laryngeal papilloma [5].

Among the 16 oncogenic types, types 16 and 18 are associated with the majority of HPV-associated cancers while types $31,33,45,52$, and 58 are responsible for $15 \%$ of cervical 
TABLE 1

\begin{tabular}{lccc}
\hline Vaccine & \multicolumn{1}{c}{ Indications } & HPV types & Contraindication \\
\hline $\begin{array}{l}\text { Cervarix/2vHPV } \\
\text { (Merck) }\end{array}$ & Cervical precancer and cancer & 16 and 18 & Latex hypersensitivity \\
\hline $\begin{array}{l}\text { Gardasil/4vHPV } \\
\text { (GlaxoSmithKline) }\end{array}$ & $\begin{array}{c}\text { Cervical cancers } \\
\text { Cervical, vaginal, vulvar, and } \\
\text { anal cancers and genital warts }\end{array}$ & $6,11,16,18$ & Yeast hypersensitivity \\
\hline $\begin{array}{l}\text { Gardasil 9/9vHPV } \\
\text { (Merck) }\end{array}$ & $\begin{array}{c}\text { Cervical, vaginal, vulvar, and } \\
\text { anal cancers and genital warts }\end{array}$ & $\begin{array}{c}6,11,16,18 \\
31,33,45,52,58\end{array}$ & \\
\hline
\end{tabular}

cancers [7]. About $91 \%$ of anal and cervical cancers, $72 \%$ of oropharyngeal cancers, $63 \%$ of penile cancers, $75 \%$ of vaginal cancers, and $69 \%$ of vulvar cancers are associated with HPV [2]. Infection with more than one HPV type at any given time is possible as $5-30 \%$ of affected individuals are infected with multiple virus types [5].

Although most infections with oncogenic HPV resolve within 1-2 years, persistent infection with an oncogenic type is necessary for the development of various HPV-associated cancers [5]. Within 5 years of infection, low-grade cervical intraepithelial neoplasia (CIN 1) may develop and potentially progress to high-grade CIN, CIN2, or CIN3 infection, leading to risk of invasive cervical cancer if left untreated [5].

Transmission occurs following minor trauma at the basal epithelium as a result of direct skin-to-skin contact during vaginal, anal, or oral sex. However, transmission can occur with nonpenetrative sexual or even nonsexual activity. Prevention of HPV by refraining from any genital contact with others or a long-term mutually monogamous relationship with an uninfected partner is the most reliable way to decrease the likelihood of transmission [5]. Correct and consistent condom use may decrease the possibility of transmission but may not provide complete protection against uncovered areas [5].

Three noninfectious, recombinant HPV vaccines are available and are prepared from type-specific HPV L1 proteins that produce type-specific virus-like particles (VLPs) [7] (see Table 1).

The quadrivalent vaccine, Gardasil, is a mixture of four HPV types, $6,11,16$, and 18, and accounts for $99 \%$ of the HPV doses distributed in the US $[1,10]$. The FDA licensed Gardasil in June 2006 for use in females aged 9-26 for prevention of cervical, vaginal, and vulvar premalignant lesions and cancers as well as anogenital warts [1]. Gardasil was later licensed in 2009 and 2010 for use in males aged 9-26 years for prevention of genital warts and the prevention of anal cancer in both males and females [30]. The bivalent vaccine, Cervarix, is a mixture of two HPV type-specific VLPs prepared from the L1 proteins of HPV types 16 and 18 [31]. Licensed in 2009, it is for use only in females aged 10-25 in the US for the prevention of cervical precancers and cancers [30]. In December 2014, the FDA licensed the 9 -valent Gardasil 9 expanding coverage of current vaccines with the addition of HPV types 31, 33, 45,52 , and 58 [7]. Gardasil 9 is for use in both sexes and incorporated into the ACIP recommendations for routine HPV vaccination in February 2015 [7].
Vaccination is considered a prophylactic measure most effective when given prior to HPV exposure [1]. The ACIP recommends vaccination starting at the age of 11-12 with either the quadrivalent, bivalent, or 9-valent vaccine for females and the quadrivalent or 9-valent vaccine for males [7]. The vaccination series is recommended for females of 13-26 and males of 13-21 years of age. Vaccine series completion is recommended through the age of 26 for immunocompromised individuals, men who have sex with men, and individuals who may already have a history of an abnormal pap test or HPV infection. The vaccine may provide additional protection from an HPV type that was not already acquired [1,7]. Vaccination is not recommended in pregnancy [7]; however pregnancy testing is not necessary prior to vaccination and no intervention is needed if administered during pregnancy. The vaccine can be coadministered with other nonlive and live vaccines in separate syringes and injection sites [32]. The dosing and administration schedules are the same for all three available vaccines and should be administered in a three-dose schedule at 0 months, 1-2 months, and 6 months [7]. Should the series be delayed or interrupted, it is not necessary to restart the schedule [7]. The ACIP recommends using the same type of HPV vaccine to complete the series to promote optimal vaccine coverage; however if the previous vaccine type is unknown, any available vaccine product can be used for series completion for females and the quadrivalent or 9-valent vaccine in males [7]. The ACIP has not yet deliberated on recommendations for 9-valent HPV vaccination administration in individuals who have completed the 3-dose series of bivalent or quadrivalent HPV 3-dose series.

3.2. Safety. Based on the evidence of clinical trials, HPV vaccination has been shown to be safe [10, 33, 34]. Among the 67 million doses of the quadrivalent vaccine distributed from 2006 to 2014 and 719,000 doses of the bivalent vaccine distributed from 2009 through 2014 in the US, 25,176 adverse events were reported [10]. Common reported adverse events were similar with all three vaccines including local injection site reactions and generalized adverse reactions of headache, fever, syncope, dizziness, and nausea $[10,35]$. Higher rates of injection site reactions have occurred with successive doses of 9-valent vaccination [36]. Additional adverse events, albeit rare, have included hospitalizations, pyrexia, allergic reaction, and asthmatic crisis [35]. Studies have found no association of quadrivalent or bivalent vaccination with 
increased risk for thromboembolic events, clotting dysfunction, anaphylaxis, hospitalizations, or emergency room visits related to autoimmune conditions, Guillain-Barre syndrome, or seizures $[34,37,38]$.

3.3. Culture. Culture supports include guidance in early discussion of the vaccine and personal beliefs in the administration of the HPV vaccine. Formal counseling of patients and parents by providers may utilize either a paternalistic or shared model for HPV vaccination presentation [21]. The paternalistic model includes vaccine recommendation as a routine vaccine while the shared model includes framing the vaccine as optional [21]. The paternalistic approach has demonstrated greater success in patient vaccine acceptance rather than differentiating HPV vaccination as separate from school-mandated vaccines [21]. Providers have noted several personal and profession supports that they consider in their own promotion of the vaccine, the most influential being a personal belief in the positive impact of the vaccine, discussing the benefits of cancer prevention and the importance of adhering to the ACIP's recommendation [24]. Providers also report that the AAP or CDC immunization recommendations are among the most influential factors for vaccine recommendations [19]. The top three HPV vaccine information sources were professional organizations (40.3\%), ACIP (31.8\%), and medical conferences (28.7\%) [39]. Additional credible resources available to healthcare providers include the following: the Immunization Action Coalition, National Network for Immunization Information, The Vaccine Education Center at The Children's Hospital of Philadelphia, and the Institute for Vaccine Safety at the Johns Hopkins Bloomberg School of Public Health.

Providers have identified additional educational interventions for patients and families such as distribution of vaccine-specific vaccine information statements (VIS) available from the CDC or other informational brochures, having pharmaceutical representative present information, and playing health information via a television in waiting rooms as helpful [22]. Providers have found strongly recommending the vaccine as safe and to be given at the age of 11-12 as well as discussing the benefits of cancer prevention often results in a positive parental response and subsequent approval for vaccination [15].

3.4. Cost and Policy. The cost of HPV vaccines is expensive, averaging $\$ 400$ for the 3 -dose series [3]. The Affordable Care Act (ACA) provisions have improved access to these vaccines, by requiring new group and healthcare plans after September, 2010, and provide no cost ACIP recommended vaccines, including HPV vaccines, to both boys and girls in the targeted age group [40]. The vaccines must be obtained, however, within their provider network [40]. Children covered by Medicaid may receive cost-free HPV vaccines until the age of 19 for males and 20 for females. The providers, however, must be a Vaccine for Children (VFC) provider to be authorized to prescribe vaccines via state laws [40].

Providers have agreed that school mandates are extremely effective at increasing vaccination rates as providers will generally offer the required school vaccines to all patients and the vaccines are usually accepted [26]. It may be beneficial and perhaps experience less opposition if all states required a 7th grade physical rather than an HPV vaccination mandate. Such a requirement would arrange providers with the opportunity to both encounter adolescents and encourage HPV vaccination at the recommended age for routine vaccination, bundling it with other recommended routine adolescent vaccinations. This strategy may allow for HPV vaccination to be generically presented, avoiding stigmatization associated with the vaccine's indication of preventing an infection that is frequently sexually transmitted [22].

3.5. Structural. Structural supports are associated with mechanisms to assist with successful HPV vaccine series initiation and completion. Providers report coadministration of HPV vaccination with other adolescent vaccinations to be beneficial in prompting remembrance to offer HPV vaccination [26]. Reminder mechanisms such as scheduling appointments for future doses at the time of the initial dose, giving the patient due date reminder cards, using reminder letters or telephone calls, and a computerized immunization information system for tracking dosage due dates are some strategies utilized to promote vaccine series completion $[17,19]$. The mail and phone reminders are often time-consuming and unsuccessful due to invalid addresses or phone numbers [22]. Providers have positively responded to the idea of using text messaging as a means of patient communication but expressed concern related to the reliability and effectiveness of this method [22]. Such simple and low-cost measures emphasizing the importance of vaccine series completion, the need to return to the clinic for future doses, and the ability of patients to correctly identify the number of remaining doses at the time of the first dose have been found to increase series completion [41]. Several professional resources and support tools are available from the CDC and pharmaceutical companies are available for providers to access a complimentary basis to help remind patients to complete the series. For instance, The Outreach Program for Vaccine Series Completion provides postagepaid postcards or recorded telephone reminder calls to the appropriate patients on behalf of providers who have enrolled in the program online. Vaccination reminder cards are also available at no charge [42].

Goal 3 of the President's Cancer Panel Annual Report for 2012-2013 is to "maximize access to HPV vaccination services." [3]. The report states that the vaccine should be available and convenient to where the adolescents receive their healthcare. Although a medical home is the preferred site, the offering of vaccines at alternative sites such as school based health centers and pharmacies has been identified as structural alternatives to vaccine series completion. A review of one school health based system found HPV vaccine completion rates to have a significantly higher $(P<0.001)$ rate of vaccine completion as compared to those of the community health centers, especially in those with low income and low insurance coverage [43]. School based health centers are often partners with community health centers, and approximately $20 \%$ receive funding through the HRSA 
Health Center Program [28]. These centers often provide services to the medically underserved and provide a trusted and safe environment for the adolescents and may provide a positive alternative to vaccine series completion [43].

Pharmacies have also been identified as a good resource to increase vaccination rates in adolescents. Pharmacies are easily accessible health professions in the US, especially in rural areas [3]. Additional advantages include longer hours of operation and no need for appointments. It is important for state mandates to develop policies for consistency in the pharmacist's authority to administer the vaccine to the targeted population, adolescents, therefore increasing access to vaccination [29].

\section{Conclusion}

Adolescent HPV vaccination coverage rates have continued to stagger despite current ACIP recommendations for routine vaccination at the age of 11-12 to prevent HPV-associated disease $[7,8]$. The estimation that coverage with at least one dose of HPV vaccine could have reached $91.3 \%$ in 2013 had these missed opportunities been eliminated shows the critical role health care providers have in optimizing HPV vaccination coverage [10]. Because providers are the gatekeepers to health information, this paper was intended to examine those provider-related factors that may influence current HPV vaccination recommendations including various knowledge, safety, cultural, cost and policy, and structural barriers. Although presented as individual barriers, many overlaps became apparent. In the various strategies addressing these barriers, it is quite evident that cost, policy, and structure are closely related. By increasing access and easing costs to the targeted population and families, it is important for both policy and structure strategies to work together to increase vaccination rates. Improved knowledge of HPV disease, transmission, vaccine safety, and recommendation, as well as the educational resources available for low or no costs, gives the provider the evidence based tools to address many of the cultural barriers identified when presenting vaccine information to parents and adolescents. It is important that all strategies continue to work in harmony to increase HPV vaccination rates in the US.

\section{Conflict of Interests}

There was no financial conflict of interests.

\section{References}

[1] L. E. Markowitz, E. F. Dunne, M. Saraiya et al., "Human papillomavirus vaccination: recommendations of the Advisory Committee on Immunization Practices (ACIP)," Morbidity and Mortality Weekly Report, vol. 63, no. RR05, pp. 1-30, 2014, http:// www.cdc.gov/mmwr/preview/mmwrhtml/rr6305al.htm.

[2] CDC, Human Papillomavirus (HPV)-Associated Cancers, CDC, 2014, http://www.cdc.gov/cancer/hpv/statistics/cases.htm.

[3] NCI, Accelerating HPV Vaccine Uptake: Urgency for Action to Prevent Cancer. A Report to the President of the United States from the President's Cancer Panel, National Cancer Institute, Bethesda, Md, USA, 2014.

[4] A. K. Chaturvedi, E. A. Engels, R. M. Pfeiffer et al., "Human papillomavirus and rising oropharyngeal cancer incidence in the United States," Journal of Clinical Oncology, vol. 29, no. 32, pp. 4294-4301, 2011.

[5] CDC, Human Papillomavirus. The Pink Book: Course Textbook, Second Printing, CDC, 12th edition, 2012, http://www.cdc.gov/ vaccines/pubs/pinkbook/hpv.html.

[6] H. W. Chesson, D. U. Ekwueme, M. Saraiya, M. Watson, D. R. Lowy, and L. E. Markowitz, "Estimates of the annual direct medical costs of the prevention and treatment of disease associated with human papillomavirus in the United States," Vaccine, vol. 30, no. 42, pp. 6016-6019, 2012.

[7] E. Petrosky, J. A. Bocchini, S. Hariri et al., "Use of 9-valent human papillomavirus (HPV) vaccine: updated HPV vaccination recommendations of the Advisory Committee on Immunization Practices," Morbidity and Mortality Weekly Report, vol. 64, no. 11, pp. 300-304, 2015.

[8] L. D. Elam-Evans, D. Yankey, J. Jeyarajah et al., "National, regional, state, and selected local area vaccination coverage among adolescents aged 13-17 years-United States, 2013," Morbidity and Mortality Weekly Report, vol. 63, no. 29, pp. 625-633, 2014.

[9] M. B. Gilkey, J. L. Moss, A.-L. McRee, and N. T. Brewer, "Do correlates of HPV vaccine initiation differ between adolescent boys and girls?" Vaccine, vol. 30, no. 41, pp. 5928-5934, 2012.

[10] S. Stokley, J. Jeyarajah, D. Yankey et al., "Human papillomavirus vaccination coverage among adolescents, 2007-2013, and postlicensure vaccine safety monitoring, 2006-2014-United States," Morbidity and Mortality Weekly Report, vol. 63, no. 29, pp. 620624, 2014.

[11] C. A. Wong, J. A. Taylor, J. A. Wright, D. J. Opel, and R. A. Katzenellenbogen, "Missed opportunities for adolescent vaccination, 2006-2011," Journal of Adolescent Health, vol. 53, no. 4, pp. 492-497, 2013.

[12] AAP, "Give a strong recommendation for HPV vaccine to increase uptake!," April 2014, http://www2.aap.org/immunization/illnesses/hpv/hpv.html.

[13] US Department of Health and Human Services, Health People 2020. Healthy People 2020 Topics and Objectives, 2014, http:// www.healthypeople.gov/2020/topics-objectives/topic/immunization-and-infectious-diseases.

[14] A. G. Litton, R. A. Desmond, J. Gilliland, W. K. Huh, and F. A. Franklin, "Factors associated with intention to vaccinate a daughter against HPV: a statewide survey in Alabama," Journal of Pediatric \& Adolescent Gynecology, vol. 24, no. 3, pp. 166-171, 2011.

[15] R. B. Perkins, J. A. Clark, G. Apte et al., "Missed opportunities for HPV vaccination in adolescent girls: a qualitative study," Pediatrics, vol. 134, no. 3, pp. e666-e674, 2014.

[16] K. R. Ylitalo, H. Lee, and N. K. Mehta, "Health care provider recommendation, human papillomavirus vaccination, and Race/Ethnicity in the US national immunization survey," American Journal of Public Health, vol. 103, no. 1, pp. 164-169, 2013.

[17] M. A. Allison, E. F. Dunne, L. E. Markowitz et al., "HPV vaccination of boys in primary care practices," Academic Pediatrics, vol. 13 , no. 5, pp. 466-474, 2013.

[18] M. F. Daley, L. A. Crane, L. E. Markowitz et al., "Human papillomavirus vaccination practices: a survey of US physicians 
18 months after licensure," Pediatrics, vol. 126, no. 3, pp. 425-433, 2010.

[19] C. C. Jim, J. Wai-Yin Lee, A. V. Groom et al., "Human papillomavirus vaccination practices among providers in Indian health service, tribal and urban Indian healthcare facilities," Journal of Women's Health, vol. 21, no. 4, pp. 372-378, 2012.

[20] M. Saraiya, J. I. Rosser, and C. P. Cooper, "Cancers that U.S. physicians believe the HPV vaccine prevents: findings from a physician survey, 2009," Journal of Women's Health, vol. 21, no. 2, pp. 111-117, 2012.

[21] C. C. Hughes, A. L. Jones, K. A. Feemster, and A. G. Fiks, "HPV vaccine decision making in pediatric primary care: a semistructured interview study," BMC Pediatrics, vol. 11, article 74, pp. 1-9, 2011.

[22] C. Gowda, S. E. Schaffer, K. J. Dombkowski, and A. F. Dempsey, "Understanding attitudes toward adolescent vaccination and the decision-making dynamic among adolescents, parents and providers," BMC Public Health, vol. 12, article 509, 10 pages, 2012.

[23] L. M. Kester, G. D. Zimet, J. D. Fortenberry, J. A. Kahn, and M. L. Shew, "A national study of HPV vaccination of adolescent girls: rates, predictors, and reasons for non-vaccination," Maternal and Child Health Journal, vol. 17, no. 5, pp. 879-885, 2013.

[24] E. L. McCave, "Influential factors in HPV vaccination uptake among providers in four States," Journal of Community Health, vol. 35, no. 6, pp. 645-652, 2010.

[25] J. A. Kahn, H. P. Cooper, S. T. Vadaparampil et al., "Human papillomavirus vaccine recommendations and agreement with mandated human papillomavirus vaccination for 11-to-12-yearold girls: a statewide survey of Texas physicians," Cancer Epidemiology Biomarkers and Prevention, vol. 18, no. 8, pp. 2325-2332, 2009.

[26] R. B. Perkins and J. A. Clark, "What affects human papillomavirus vaccination rates? A qualitative analysis of providers' perceptions," Women's Health Issues, vol. 22, no. 4, pp. e379e386, 2012.

[27] J. L. Moss, A. L. Feld, B. O’Malley et al., “Opportunities for increasing human papillomavirus vaccine provision in school health centers," Journal of School Health, vol. 84, no. 6, pp. 370378, 2014.

[28] US Department of Health and Human Services, Health Resources and Services Administration, 2015, http://www.hrsa .gov/ourstories/schoolhealthcenters.

[29] N. T. Brewer, J. K. Chung, H. M. Baker, M. C. Rothholz, and J. S. Smith, "Pharmacist authority to provide HPV vaccine: novel partners in cervical cancer prevention," Gynecologic Oncology, vol. 132, supplement 1, pp. S3-S8, 2014.

[30] CDC, "FDA licensure of quadrivalent human papillomavirus vaccine (HPV4, Gardisil) for use in males and guidance from the Advisory Committee on Immunization Practices (ACIP)," Morbidity and Mortality Weekly Report, vol. 59, no. 20, pp. 630632, 2010.

[31] CDC, "FDA licensure of bivalent human papillomavirus vaccine (HPV2, Cervarix) for use in females and updated HPV vaccination recommendations from the Advisory Committee on Immunization Practices (ACIP)," Morbidity and Mortality Weekly Report, vol. 59, no. 20, pp. 626-629, 2010.

[32] A. S. Noronha, L. E. Markowitz, and E. F. Dunne, "Systematic review of human papillomavirus vaccine coadministration," Vaccine, vol. 32, no. 23, pp. 2670-2674, 2014.

[33] N. P. Klein, J. Hansen, C. Chao et al., "Safety of quadrivalent human papillomavirus vaccine administered routinely to females," Archives of Pediatrics and Adolescent Medicine, vol. 166, no. 12, pp. 1140-1148, 2012.

[34] P. S. Sow, D. Watson-Jones, N. Kiviat et al., "Safety and immunogenicity of human papillomavirus-16/18 AS04-adjuvanted vaccine: a randomized trial in 10-25-year-old HIV-seronegative African girls and young women," The Journal of Infectious Diseases, vol. 207, no. 11, pp. 1753-1763, 2013.

[35] FDA, "Highlights of prescribing information. Gardasil 9 (human papillomavirus 9-valent vaccine, recombinant)," 2014, http://www.fda.gov/BiologicsBloodVaccines/Vaccines/ApprovedProducts/ucm426520.htm.

[36] E. A. Joura, A. R. Giuliano, O.-E. Iversen et al., "A 9-valent HPV vaccine against infection and intraepithelial neoplasia in women," The New England Journal of Medicine, vol. 372, no. 8, pp. 711-723, 2015.

[37] FDA, Product approval-prescribing information (package insert). Cervarix[human papillomavirus bivalent (types 16 and 18) vaccine, recombinant], GlaxoSmithKline Biologicals, FDA, 2009, http://www.fda.gov/downloads/BiologicsBloodVaccines/ Vaccines/ApprovedProducts/UCM186981.pdf.

[38] J. Gee, A. Naleway, I. Shui et al., "Monitoring the safety of quadrivalent human papillomavirus vaccine: findings from the Vaccine Safety Datalink," Vaccine, vol. 29, no. 46, pp. 8279-8284, 2011.

[39] S. T. Vadaparampil, J. A. Kahn, D. Salmon et al., "Missed clinical opportunities: provider recommendations for HPV vaccination for 11-12 year old girls are limited," Vaccine, vol. 29, no. 47, pp. 8634-8641, 2011.

[40] U.S. Department of Health and Human Services, "The affordable care act and immunization," August 2015, http://www .hhs.gov/healthcare/facts/factsheets/2010/09/The-AffordableCare-Act-and-Immunization.html.

[41] R. Gold, A. Naleway, and K. Riedlinger, "Factors predicting completion of the human papillomavirus vaccine series," Journal of Adolescent Health, vol. 52, no. 4, pp. 427-432, 2013.

[42] Merck \& Co Inc, MerkVaccines.com: Professional Resources, Support, \& More, 2014, https://www.merckvaccines.com/Professional-Resources/Pages/ProfessionalResourcesHome?WT.svl=5.

[43] S. G. Federico, L. Abrams, R. M. Everhart, P. Melinkovich, and S. J. Hambidge, "Addressing adolescent immunization disparities: a retrospective analysis of school-based health center immunization delivery," American Journal of Public Health, vol. 100, no. 9, pp. 1630-1634, 2010. 

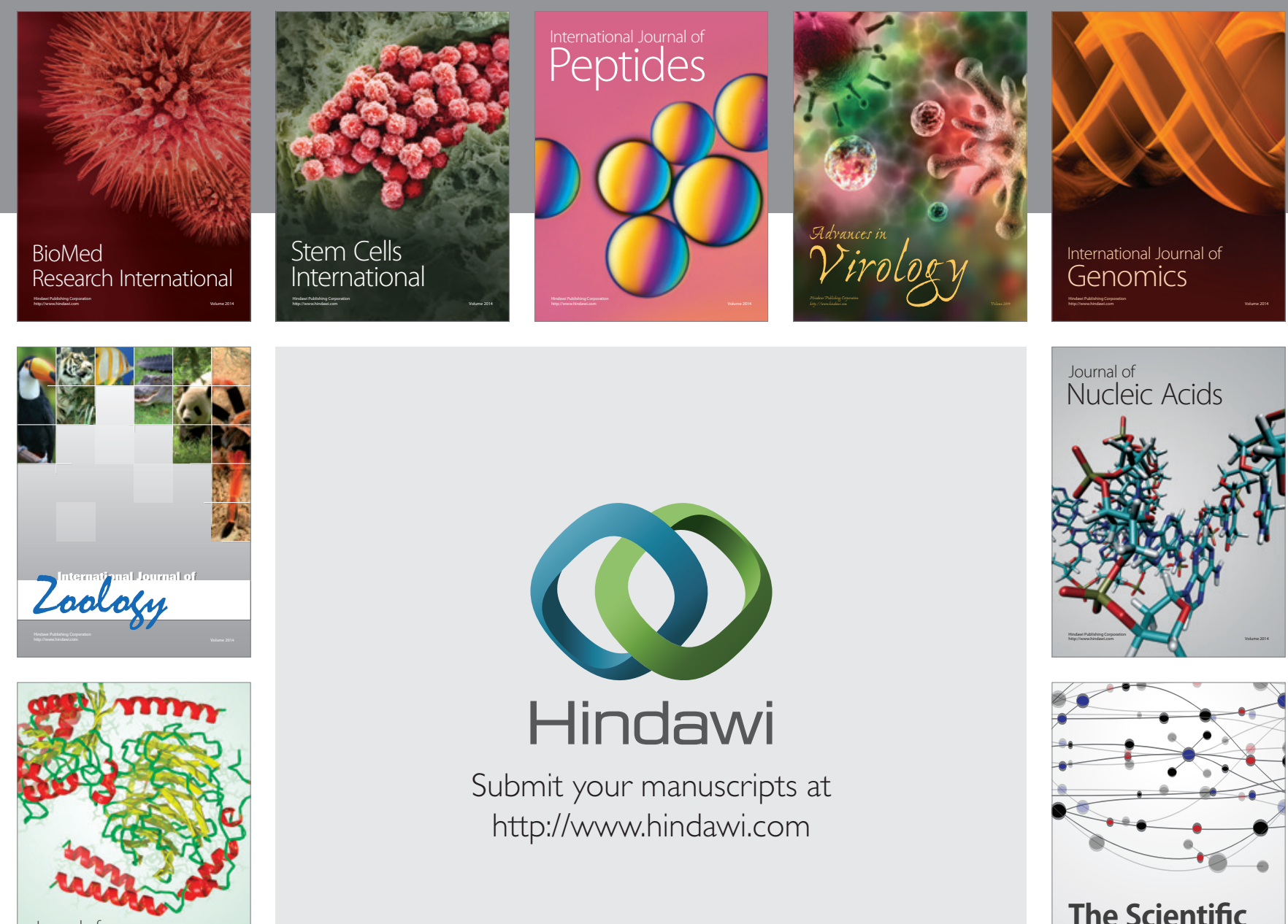

Submit your manuscripts at

http://www.hindawi.com

Journal of
Signal Transduction
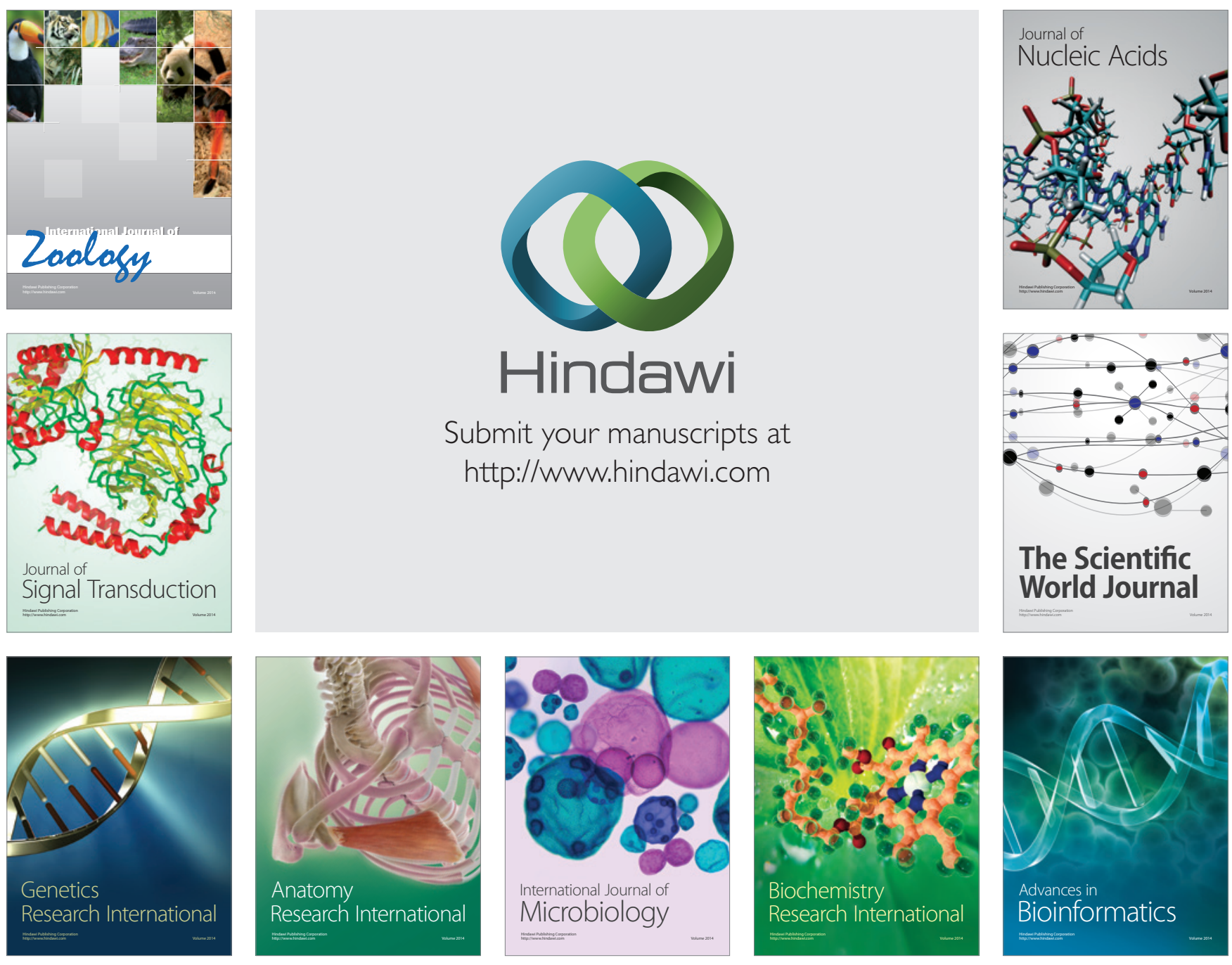

The Scientific World Journal
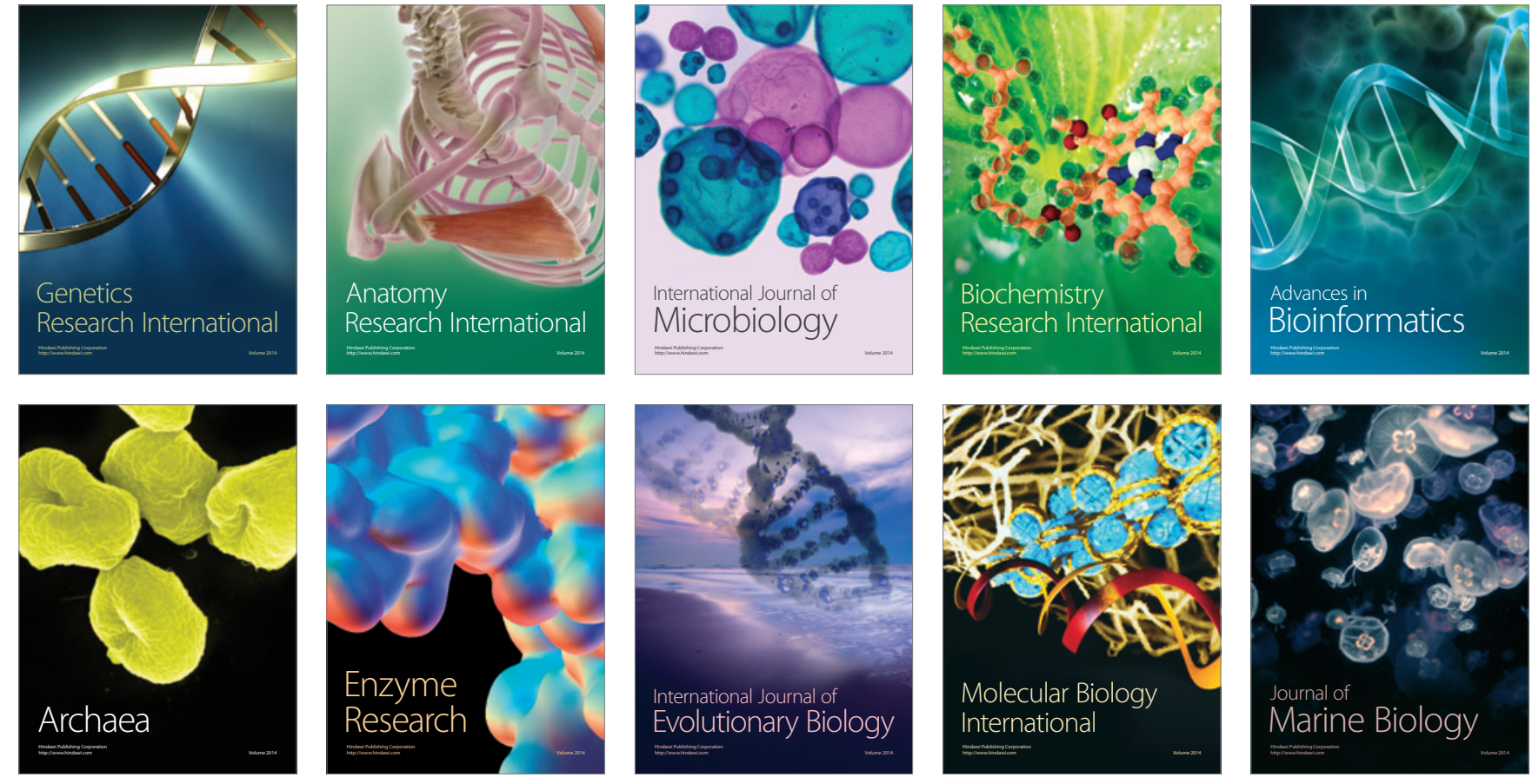